\section{Suicide in India}

We read with interest the editorial on suicide prevention from a life course perspective (Gunnell \& Lewis, 2005). It offers a broad conceptual overview of the issues related to suicide.

Recent reports from Vellore suggest that suicide rates in India are grossly underreported (Joseph et al, 2003; Aaron et al, 2004; Abraham et al, 2005; Prasad et al, 2005). The average annual suicide rate was 95 per 100000 for the years 1994-99. The rates in adolescent males and females and those over 55 years were 148, 58 and 189 per 100000 respectively. Data from India on the contribution of mental illness to suicide rates are limited. A study from Chennai reported a higher risk of mental disorder among people who die by suicide compared with controls (Vijaykumar \& Rajkumar, 1999). However, other evidence suggests that chronic stress and precipitating life events rather than severe mental disorders are the major risk factors for suicide. Recent adverse life events, interpersonal stress and relationship difficulties, severe financial distress, the use of alcohol and issues related to gender have all been associated with suicide (Prasad et al, 2005). The depiction of suicide in the mass media is also contributory. Last but not least is the fact that many people seem to accept suicide as an option when faced with extreme mental distress.

Although psychiatric disorders are often associated with suicide in the West and medical models are employed, in developing countries social, economic and cultural factors must be considered when attempting to explain the persistently high rates, the impulsive and stress-related deaths and the apparent widespread 'acceptability' of such an option in society. Considering suicide as a single phenomenon or even as a single final pathway might be simplistic. Many diverse approaches, tailored to regional factors, will have to be implemented simultaneously to produce any global reduction in suicide rates.

Aaron, R., Joseph, A., Abraham, S., et al (2004) Suicides in young people in rural southern India. Lancet, 363, $1117-1118$.

Abraham, V. J., Abraham, S. \& Jacob, K. S. (2005) Suicide in the elderly in Kaniyambadi block, Tamil Nadu, South India. International journal of Geriatric Psychiatry, 20, 953-955.
Gunnell, D. \& Lewis, G. (2005) Studying suicide from the life course perspective: implications for prevention. British Journal of Psychiatry, 187, 206-208.

Joseph, A., Abraham, S., Muliyil, J. P., et al (2003) Evaluation of suicide rates in rural India using verbal autopsies, 1994-99. BM], 326, ||2|-|122.

Prasad, J., Abraham, V. J., Minz, S., et al (2005) Rate and factors associated with suicide in Kaniyambadi Block, Tamil Nadu, South India, 2000-02. International Journal of Social Psychiatry, in press.

Vijaykumar, L. \& Rajkumar, S. (1999) Are risk factor for suicide universal? A case-control study in India. Acto Psychiatrica Scandinavica, 99, 407-4II.

S. D. Manoranjitham, R. Jayakaran, K. S.

Jacob Christian Medical College, Vellore 63202, India. E-mail: ksjacob@cmcvellore.ac.in

\section{Metabolic syndrome and schizophrenia}

In his recent editorial Dr Thakore (2005) rightly highlights the importance of the association of the metabolic syndrome and one of its consequences, type 2 diabetes, with schizophrenia. Despite acknowledging that antipsychotic drugs can induce substantial weight gain, he avoids ascribing the metabolic disturbances in schizophrenia to drug-induced obesity. His suggestion that untreated schizophrenia is itself associated with metabolic disturbance is based on a study of 19 people who had substantially greater deposits of intra-abdominal fat than a control group (Ryan et al, 2004). This contrasts with other studies showing that 40 antipsychotic-naïve patients with schizophrenia had no elevation in intra-abdominal fat compared with controls (Zhang et al, 2004) and that 50 did not differ from a control group in terms of body mass index, fasting plasma glucose or insulin (Arranz et al, 2004). In attempting to explain discrepancies in terms of methodological differences, Dr Thakore is wrong to state that the control group of Zhang et al consisted of 'elderly men'; controls were well matched for age and gender with the patient group.

These larger studies also show that antipsychotic drug treatment is associated with increased intra-abdominal fat (Zhang et al, 2004) and insulin resistance (Arranz et al, 2004), despite negative findings from Ryan et al (2004). The risk of diabetes in schizophrenia is higher in patients receiving olanzapine rather than conventional antipsychotics (Koro et al, 2002); olanzapine is particularly liable to induce weight gain. These and other studies indicate that antipsychotic drug treatment can result in metabolic morbidity. It would thus be misleading, if not dangerous, to imply that obesity resulting from treatment with some antipsychotic drugs is not associated with the development of the metabolic syndrome and type 2 diabetes.

Dr Thakore listed criteria for the metabolic syndrome; these have now been superseded by a more clinically accessible and less stringent definition. The core criterion is central (abdominal) obesity, defined by waist circumference, plus two of four risk factors from elevated triglycerides, reduced high-density lipoprotein cholesterol, raised blood pressure and raised fasting plasma glucose (International Diabetes Federation, 2005).

\section{Declaration of interest}

G.P.R. has received research support and honoraria from Bristol-Myers Squibb and Janssen-Cilag.

Arranz, B., Rosel, P., Ramirez, N., et al (2004) Insulin resistance and increased leptin concentrations in noncompliant schizophrenia patients but not in antipsychotic-naive first-episode schizophrenia patients. Journal of Clinical Psychiatry, 65, 1335-1342.

International Diabetes Federation (2005) The IDF

Consensus Worldwide Definition of the Metabolic Syndrome. http: //www.idf.org/webdata/docs/ Meta_syndrome_def.pdf

Koro, C. E., Fedder, D. O., L'Italien, G. J., et al (2002) Assessment of independent effect of olanzapine and risperidone on risk of diabetes among patients with schizophrenia: population based nested case-control study. BMJ, 325, 243-247.

Ryan, M. C. M., Flanagan, S., Kinsella, U., et al (2004) Atypical antipsychotics and visceral fat distribution in first episode, drug-naive patients with schizophrenia. Life Sciences, 74, 1999-2008.

Thakore, J. H. (2005) Metabolic syndrome and schizophrenia. British Journal of Psychiatry, 186, 455-456.

Zhang, Z.-J., Yao, Z.-J., Liu, W., et al (2004) Effects of antipsychotics on fat deposition and changes in leptin and insulin levels. British Journal of Psychiatry, 184, 58-62.

G. P. Reynolds Division of Psychiatry and Neuroscience, Queen's University Belfast, Whitla Medical Building, 97 Lisburn Road, Belfast BT9 7BL, UK.E-mail: g.reynolds@qub.ac.uk

Author's reply: Critical methodological differences between the studies of Zhang et al (2004) and Ryan et al (2004) might explain why the two fail to agree. Zhang et al reported a standard deviation of 50 for the age of the controls, indicating that some were elderly; moreover the groups were not matched for gender. This is important as elderly males have higher amounts of intra-abdominal fat (IAF). Lifestyle parameters such as diet, exercise, 